\title{
Hyperon-nucleon interaction in chiral effective field theory
}

\author{
Johann Haidenbauer \\ Institute for Advanced Simulation, Forschungszentrum Jülich GmbH, D-52425 Jülich, Germany
}

DOI: http://dx.doi.org/10.3204/DESY-PROC-2014-04/134

\begin{abstract}
Results of a study of the hyperon-nucleon system within chiral effective field theory are reported. The investigation is based on the scheme proposed by Weinberg which has been applied rather successfully to the nucleon-nucleon interaction in the past. Results for the $\Lambda N$ and $\Sigma N$ interactions in free space and nuclear matter, obtained up to next-to-leading order, are presented and discussed.
\end{abstract}

\section{Introduction}

Chiral effective field theory (EFT) as proposed in the pioneering works of Weinberg [1] is a powerful tool for the derivation of baryonic forces. In this scheme there is an underlying power counting which allows to improve calculations systematically by going to higher orders in a perturbative expansion. In addition, it is possible to derive two- and corresponding three-body forces as well as external current operators in a consistent way.

Recently, a hyperon-nucleon $(Y N)$ interaction has been derived up to next-to-leading order (NLO) in chiral EFT by the Jülich-Bonn-Munich group [2]. At that order there are contributions from one- and two-pseudoscalar-meson exchange diagrams and from four-baryon contact terms without and with two derivatives. $\mathrm{SU}(3)$ flavor symmetry is imposed for constructing the $Y N$ interaction in order to reduce the number of free parameters, in particular the number of lowenergy constants (LECs) associated with the arising contact terms. In the actual calculation the $\mathrm{SU}(3)$ symmetry is broken, however, by the mass differences between the involved mesons $(\pi, K, \eta)$ and between the baryons $(N, \Lambda, \Sigma)$.

An excellent description of available $\Lambda N$ and $\Sigma N$ scattering data could be achieved at NLO. Corresponding results are reported in Sect. 3. In addition the in-medium properties of the EFT $Y N$ interaction have been investigated. Specifically, binding energies of the $\Lambda$ and $\Sigma$ hyperons in nuclear matter have been calculated, based on a conventional first-order Brueckner calculation, and these are also presented.

\section{The $Y N$ interaction in chiral EFT}

The derivation of the chiral baryon-baryon potentials for the strangeness sector at leading order (LO) using the Weinberg power counting is outlined in Refs. [3, 4]. Details for the NLO case can be found in Ref. [2], see also [5]. The LO potential consists of four-baryon contact terms without derivatives and of one-pseudoscalar-meson exchanges while at NLO contact terms with 
two derivatives arise, together with contributions from (irreducible) two-pseudoscalar-meson exchanges. The contributions from pseudoscalar-meson exchanges $(\pi, \eta, K)$ are completely fixed by the assumed $\mathrm{SU}(3)$ flavor symmetry. On the other hand, the strength parameters associated with the contact terms, the LECs, need to be determined in a fit to data. How this is done is described in detail in Ref. [2]. Note that we impose also SU(3) symmetry for those contact terms which reduces the number of independent LECs that can contribute.

The reaction amplitudes are obtained from the solution of a coupled-channels LippmannSchwinger (LS) equation for the interaction potentials:

$$
\begin{aligned}
T_{\nu^{\prime \prime} \nu^{\prime}}^{\rho^{\prime \prime} \rho^{\prime}, J}\left(p^{\prime \prime}, p^{\prime} ; \sqrt{s}\right)= & V_{\nu^{\prime \prime} \nu^{\prime}}^{\rho^{\prime \prime} \rho^{\prime}, J}\left(p^{\prime \prime}, p^{\prime}\right)+ \\
& \sum_{\rho, \nu} \int_{0}^{\infty} \frac{d p p^{2}}{(2 \pi)^{3}} V_{\nu^{\prime \prime} \nu}^{\rho^{\prime \prime} \rho, J}\left(p^{\prime \prime}, p\right) \frac{2 \mu_{\nu}}{q_{\nu}^{2}-p^{2}+i \eta} T_{\nu \nu^{\prime}, J}^{\rho \rho^{\prime}, J}\left(p, p^{\prime} ; \sqrt{s}\right) .
\end{aligned}
$$

The label $\nu$ indicates the particle channels and the label $\rho$ the partial wave. $\mu_{\nu}$ is the pertinent reduced mass. The on-shell momentum in the intermediate state, $q_{\nu}$, is defined by $\sqrt{s}=\sqrt{m_{B_{1, \nu}}^{2}+q_{\nu}^{2}}+\sqrt{m_{B_{2, \nu}}^{2}+q_{\nu}^{2}}$. Relativistic kinematics is used for relating the laboratory energy $T_{\text {lab }}$ of the hyperons to the c.m. momentum.

We solve the LS equation in the particle basis, in order to incorporate the correct physical thresholds. Depending on the total charge, up to three baryon-baryon channels can couple. The Coulomb interaction is taken into account appropriately via the Vincent-Phatak method [6]. The potentials in the LS equation are cut off with a regulator function, $f_{R}(\Lambda)=$ $\exp \left[-\left(p^{\prime 4}+p^{4}\right) / \Lambda^{4}\right]$, in order to remove high-energy components [7]. We consider cutoff values in the range $\Lambda=500-650 \mathrm{MeV}$, similar to what was used for chiral $N N$ potentials [7].

\section{Results for $\Lambda N$ and $\Sigma N$ in free space and nuclear matter}

Our results for $\Lambda N$ and $\Sigma N$ scattering are presented in Fig. 1. The bands (black/red for NLO and grey/green bands for LO) represent the variation of the cross sections based on chiral EFT within the considered cutoff region, i.e. $550-700 \mathrm{MeV}$ in the LO case [3] and 500-650 MeV at NLO. For comparison also results for the Jülich '04 [8] meson-exchange model are shown (dashed line).

Obviously, the available $\Lambda N$ and $\Sigma N$ scattering data are very well described by our NLO EFT interaction. In particular, and as expected, the energy dependence exhibited by the data is visibly better reproduced within our NLO calculation than at LO. This concerns in especially the $\Sigma^{+} p$ channel. But also for $\Lambda p$ the NLO results are now well in line with the data even up to the $\Sigma N$ threshold. Furthermore, one can see that the dependence on the cutoff mass is strongly reduced in the NLO case. Additional results, for differential cross sections and for phase shifts, can be found in Ref. [2].

Besides an excellent description of the $Y N$ data the chiral EFT interaction yields a satisfactory value for the hypertriton binding energy, see Ref. [2]. Calculations for the four-body hypernuclei ${ }_{\Lambda}^{4} \mathrm{H}$ and ${ }_{\Lambda}^{4} \mathrm{He}$ based on the EFT interactions can be found in Ref. [9].

Recently, we have also investigated the properties of our $Y N$ interactions in nuclear matter [10]. Specifically, we performed a conventional first-order Brueckner calculation based on the standard choice of the single-particle (s.p.) potentials. Table 3 summarizes preliminary results for the $\Lambda$ and $\Sigma$ potential depths, $U_{\Lambda}\left(p_{\Lambda}=0\right)$ and $U_{\Sigma}\left(p_{\Sigma}=0\right)$, evaluated at the saturation point of nuclear matter, i.e. for $k_{F}=1.35 \mathrm{fm}^{-1}$. Corresponding results obtained for the Jülich 


\section{HYPERON-NUCLEON INTERACTION IN CHIRAL EFFECTIVE FIELD THEORY}
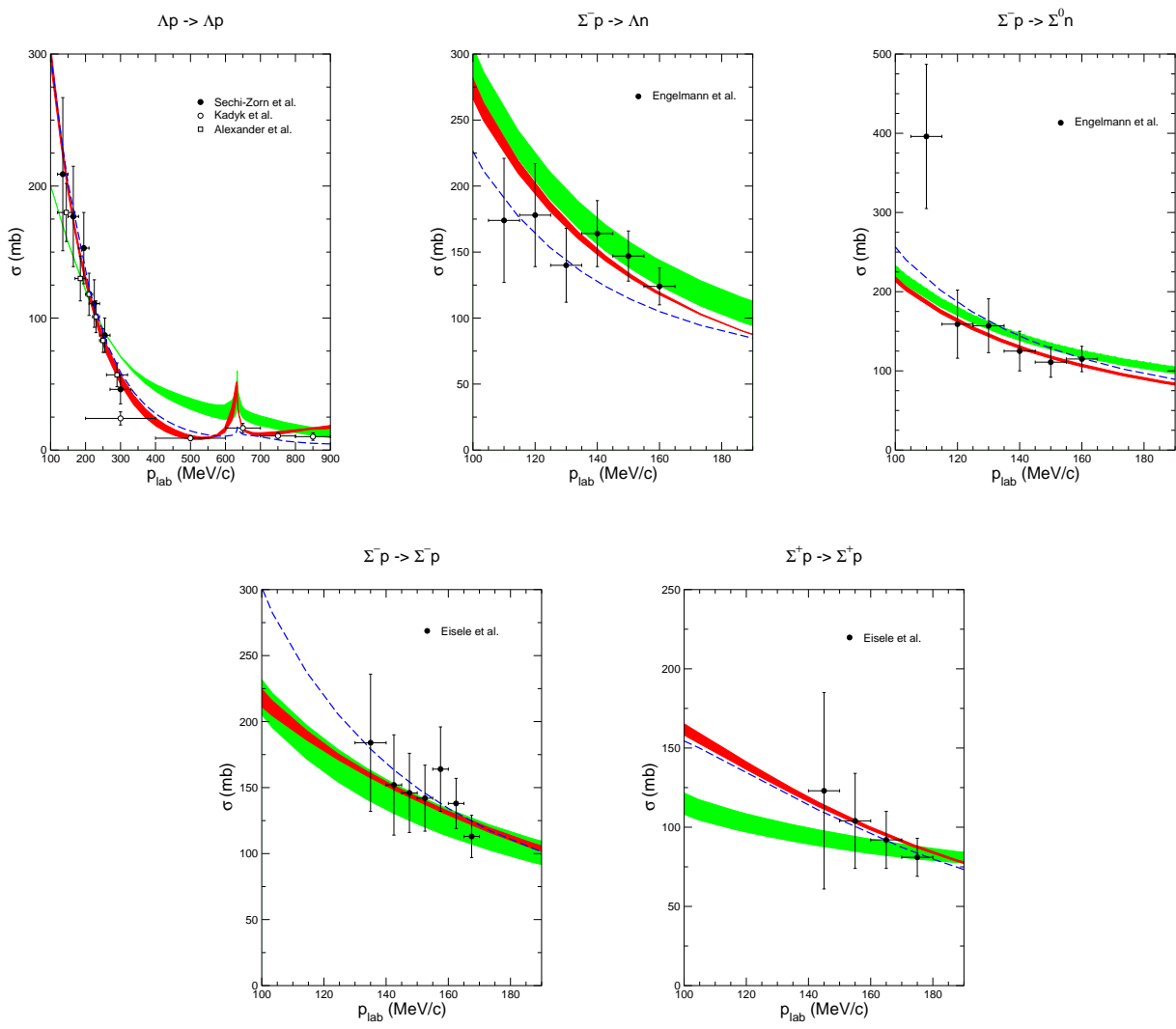

Figure 1: Total cross sections for $\Lambda N$, and $\Sigma N$ scattering as a function of $p_{l a b}$. The grey/green band shows the chiral EFT results to LO for variations of the cut-off in the range $\Lambda=550$ $700 \mathrm{MeV}$, while the black/red band are results to NLO for $\Lambda=500-650 \mathrm{MeV}$. The dashed curve is the result of the Jülich '04 [8] meson-exchange potential.

meson-exchange potentials from 2004 [8] and 1994 [11] are also included. In case of the EFT results we show the variation with the cutoff. These are comparable for $U_{\Lambda}$ at LO and NLO, but noticeably reduced for $U_{\Sigma}$ at NLO. The predictions for $U_{\Lambda}(0)$ are well in line with the 'empirical' value for the $\Lambda$ binding energy in nuclear matter of about -27 to $-30 \mathrm{MeV}$, deduced from the binding energies of finite $\Lambda$ hypernuclei [12].

As already emphasized in Ref. [2] the interaction in the ${ }^{3} S_{1}$ partial wave of the $\Sigma^{+} p$ channel is repulsive, for the LO potential but also for the NLO interaction. As a consequence the predicted $\Sigma$ s.p. potential is likewise repulsive, see Table 3. Such a repulsion is in accordance with evidence from the analysis of level shifts and widths of $\Sigma^{-}$atoms and from recently measured $\left(\pi^{-}, K^{+}\right)$inclusive spectra related to $\Sigma^{-}$-formation in heavy nuclei $[13,14]$. 
Table 1: Results for the s.p. potentials $U_{\Lambda}(0)$ and $U_{\Sigma}(0)$ (in $\mathrm{MeV}$ ) based on our EFT interactions and the Jülich meson-exchange interactions.

\begin{tabular}{|c||r|r|r|r|}
\hline & EFT LO & EFT NLO & Jülich '04 [8] & Jülich '94 [11] \\
\hline$\Lambda[\mathrm{MeV}]$ & $550 \cdots 700$ & $500 \cdots 650$ & & \\
\hline$U_{\Lambda}(0)$ & $-38.0 \cdots-34.4$ & $-29.3 \cdots-22.9$ & -51.2 & -29.8 \\
\hline$U_{\Sigma}(0)$ & $28.0 \cdots 11.1$ & $17.4 \cdots 12.1$ & -22.2 & -71.45 \\
\hline
\end{tabular}

\section{Summary}

Chiral effective field theory, successfully applied in Ref. [7] to the $N N$ interaction, also works well for the baryon-baryon interactions in the strangeness sector $[2,15,16]$. In particular, the results for the $Y N$ interaction presented here, obtained to next-to-leading order in the Weinberg counting, are very encouraging. First there is a visible improvement in the quantitative reproduction of the available data on $\Lambda N$ and $\Sigma N$ scattering and, secondly, the dependence on the regularization scheme is strongly reduced as compared to the LO result. Indeed the description of the $Y N$ system achieved at NLO is now on the same level of quality as the one by the most advanced meson-exchange $Y N$ interactions.

\section{Acknowledgements}

I would like to thank N. Kaiser, U.-G. Meißner, A. Nogga, S. Petschauer, and W. Weise for collaborating on the topics covered by my talk. Work supported in part by DFG and NSFC (CRC 110).

\section{References}

[1] S. Weinberg, Phys. Lett. B 251 (1990) 288.

[2] J. Haidenbauer, S. Petschauer, N. Kaiser, U.-G. Meißner, A. Nogga, W. Weise, Nucl. Phys. A 91524 (2013).

[3] H. Polinder, J. Haidenbauer, U.-G. Meißner, Nucl. Phys. A 779244 (2006).

[4] J. Haidenbauer, U.-G. Meißner, A. Nogga, H. Polinder, Lect. Notes Phys. 724113 (2007).

[5] S. Petschauer, N. Kaiser, Nucl. Phys. A 9161 (2013).

[6] C.M. Vincent, S.C. Phatak, Phys. Rev. C 10391 (1974).

[7] E. Epelbaum, W. Glöckle, U.-G. Meißner, Nucl. Phys. A 747362 (2005).

[8] J. Haidenbauer, U.-G. Meißner, Phys. Rev. C 72044005 (2005).

[9] A. Nogga, Few Body Syst. 55, 757 (2014).

[10] J. Haidenbauer, U.-G. Meißner, Nucl. Phys. A 93629 (2015).

[11] A. Reuber, K. Holinde, J. Speth, Nucl. Phys. A 570543 (1994).

[12] D.J. Millener, C.B. Dover, A. Gal, Phys. Rev. C 382700 (1988).

[13] M. Kohno, Y. Fujiwara, Y. Watanabe, K. Ogata and M. Kawai, Phys. Rev. C 74064613 (2006).

[14] J. Dabrowski and J. Rozynek, Phys. Rev. C 78037601 (2008).

[15] H. Polinder, J. Haidenbauer, U.-G. Meißner, Phys. Lett. B 65329 (2007).

[16] J. Haidenbauer, U.-G. Meißner, Phys. Lett. B 684275 (2010). 